\title{
Can useful toroidal current be driven by classical viscoresistive Alfvén waves?
}

\author{
P. M. Bellan and M. A. Schalita) \\ California Institute of Technology, Pasadena, California 91125
}

(Received 18 July 1990; accepted 12 October 1990)

\begin{abstract}
Simple, yet exact, analytic solutions for the shear and compressional Alfvén wave are obtained for helical magnetohydrodynamic (MHD) waves in cylindrical geometry with both resistivity and viscosity included. The current driven by all possible combinations of these waves is examined in the quasilinear regime (i.e., where the magnetic field produced by the driven current is not self-consistently included in the equilibrium where the wave equations are derived). It is found in all cases that it is not possible to drive significant bulk axial current with small amplitude wave fields. Thus, any useful low-frequency current drive scheme will have to be based on phenomena more complicated than those discussed here.
\end{abstract}

\section{INTRODUCTION}

Tokamaks work by having a large toroidal current produce the nested flux surfaces necessary for particle confinement. The conventional method for driving this current, induction (i.e., transformer action), has the drawback of being inherently pulsed. Since steady-state operation would be desirable for a fusion reactor, there is considerable interest in finding ways to drive steady-state toroidal currents. Steadystate toroidal current by high-frequency plasma waves (e.g., lower hybrid, ' electron cyclotron, ${ }^{2}$ fast mode ${ }^{3}$ ) has been amply demonstrated and is reasonably well understood.

There have recently been investigations into the possibility of toroidal current drive by low-frequency waves and several schemes have been proposed. ${ }^{4-7}$ Low frequency has three possible advantages compared to high frequency: (i) the technology is likely to be significantly less expensive, (ii) the current drive efficiency may be higher, and (iii) the problems of wave penetration into the plasma may bc lcss severe.

Let us now briefly review the various low-frequency schemes that have been discussed. Ohkawa ${ }^{4}$ proposed a simple scheme involving circularly polarized shear Alfvén waves with no radial dependence, but with damping in the axial direction, i.e., the axial wave vector $k$ was complex. Mett and Tataronis ${ }^{5}$ also considered shear Alfvén waves, and also had no radial dependence, but assumed tha $k$ was real and invoked viscosity as well as resistivity. Taylor ${ }^{6}$ considered shear Alfvén waves with complex $k$, invoked viscosity, and again had no radial dependence. Chan, Miller, and Ohkawa' considered the general case of complex axial and radial wave vectors and examined current drive by both shear and compressional modes as well as the kinetic effect of enhanced damping by minority ion cyclotron resonance.

In addition to the Alfvén schemes discussed above, there also exist schemes based on the concept of ac magnetic helicity injection. ${ }^{8-11}$ The theory of these helicity injection schemes sidesteps describing the detailed physical phenomena providing the current drive, but instead is based on the concept that injecting magnetic helicity is equivalent to driv-

\footnotetext{
" Present address: Hughes Aircraft Space and Communications Group, El Segundo, California 90009.
}

ing force-free current. The requirements for injecting helicity are prescribed by a simple helicity conservation equation $^{8}$ easily derived from Faraday's law and Ohm's law. Since the helicity schemes involve the same magnetohydrodynamic (MHD) equations as the Alfvén schemes, it is presumed that there is a close relation between the two schemes. In effect, the helicity approach provides a zero-dimensional, conservation-type description, whereas the Alfvén approach describes the detailed dynamics. For example. Ohkawa ${ }^{4}$ and also Chan, Miller, and Ohkawa ${ }^{7}$ interpreted some of their schemes in terms of helicity injection. However, Bellan ${ }^{12}$ and Schalit and Bellan ${ }^{13}$ showed that the helicity injection point of view may be complicated by the fact that there can also exist significant dissipation of helicity by the oscillating fields themselves, so that injected helicity might be dissipated entirely by the oscillating ficlds rather than providing the desired dc current drive. Taylor ${ }^{6}$ also discussed the importance of helicity dissipation by oscillating fields.

There have been no conclusive experimental demonstrations of Alfvén wave current drive injection, except possibly for a very small experiment by Hotta et al. ${ }^{14}$ Experiments by Schoenberg et al..$^{15}$ and by Bellan ${ }^{16}$ to test ac helicity injection failed to demonstrate any conclusive current drive.

Therc also cxists another class of low-frequency current drive schemes, namely rotamaks, ${ }^{17-20}$, rhythmacs, ${ }^{21}$ and their predecessors. ${ }^{22-24}$ Unlike the Alfvén schemes, in rotamaks and rhythmacs the ions are assumed stationary, the oscillating field is a forced oscillation rather than a wave, and current drive is explained in terms of a Hall term in the Ohm's law and a well understood, ${ }^{18}$ highly nonlinear anomalous skin effect. The nonlinear skin effect results from the fact that electrons, trapped in a traveling magnetic field, see the frequency of the traveling field Doppler shifted down to zero, and so cannot shield the traveling field because in the electron frame this field appears to be static. The Alfven schemes, in contrast, invoke neither the Hall term nor the anomalous skin effect. While rotamak and rhythmac experiments have demonstrated significant current drive, the plasmas involved were always cold and incompletely ionized. The Hotta experiment ${ }^{14}$ may fall into the rotamak class even though it was interpreted as an Alfvén drive. 
In this paper we will consider current drive by classical Alfvén waves in cylindrical geometry with both viscosity and resistivity included. We will not include Hall terms or anomalous skin effects. Because of the toroidal periodicity of a tokamak, the toroidal wave vector must always be real and any dissipation must be manifested by a radial damping. We will approximate the toroidal geometry by a straight cylinder with coordinates $r, \theta, z$. Thus, the periodicity condition on the toroidal wave vector translates to the condition that the axial wave vector $k$ be real, and a toroidal mode number $n$ corresponds to $k=n / R$, where $R$ is the tokamak major radius. (Note that, in contrast, Ohkawa ${ }^{4}$ and Taylor ${ }^{6}$ assume a complex $k$, inconsistent with toroidal periodicity.)

The main result of our analysis is that while it is possible to drive bulk current drive with classical Alfvén waves having weak radial damping, this current drive is impractical because the required amplitudes of oscillating fields are unrealistically large. This result complements (i.e., is consistent with) the results of Chan, Miller, and Ohkawa, ${ }^{7}$ who found that efficient current drive was possible with classical Alfvén waves having strong radial damping, but that this current drive was impractical because only a surface current was driven.

\section{DERIVATION OF VISCORESISTIVE ALFVÉN WAVES IN CYLINDRICAL GEOMETRY}

To be useful, a current drive scheme must be able to drive bulk current all the way from the edge to the magnetic axis of the tokamak; schemes that simply drive surface currents are of no interest. Thus, our goal is to find a method that will drive such bulk currents, which in the straight cylinder approximation corresponds to currents that are finite at $r=0$ and that have a weak $r$ dependence. Because of the peculiar nature of azimuthal Fourier expansions, only modes with $m=1$ are finite ${ }^{25}$ at $r=0$, so that our investigation will focus on the behavior of $m=1$ cylindrical modes in the neighborhood of $r=0$. Initially, however, we will let $m$ be an arbitrary integer.

We consider Fourier modes $\sim \exp (i m \theta+i k z)$. Upper case letters denote equilibrium quantities and lower case denote fluctuating quantities. The cylindrical geometry suggests that our solutions should be in the form of Bessel functions, and defining $s=k r$ we note the useful identity

$$
\nabla^{2} J_{m}(\gamma s)=-k^{2}\left(1+\gamma^{2}\right) J_{m}(\gamma s),
$$

where $\gamma$ is arbitrary.

Before launching into the wave physics, we note that the magnetic field must always satisfy

$$
\boldsymbol{\nabla} \cdot \mathbf{b}=0
$$

There are two general polarizations of $\mathbf{b}$ that satisfy Eq. (2), namely, $\mathbf{b}=\nabla z \times \nabla \psi$ and $\mathbf{b}=\nabla \times(\nabla \mathbf{z} \times \nabla \chi)$, where $\psi$ and $\chi$ are arbitrary scalar functions. Following Moffatt's $\mathrm{s}^{26}$ terminology, the former polarization is called a toroidaltype field, while the latter is called a poloidal-type field. Using Bessel functions as the scalar functions, the two polarizations can be written as

$$
\begin{aligned}
\mathbf{b}_{c} & =-\left(b_{z} / \gamma^{2} k^{2}\right) \nabla \times\left[\hat{z} \times \nabla J_{m}(\gamma s)\right] \\
& =\left(b_{z} / i \gamma^{2} k\right)\left[i k\left(1+\gamma^{2}\right) J_{m}(\gamma s) \hat{z}-\nabla J_{m}(\gamma s)\right]
\end{aligned}
$$

and

$$
\mathbf{b}_{s}=\left(i b_{1} / k \beta\right) \hat{z} \times \nabla J_{m}(\beta s)
$$

where $b_{z}$ and $b_{1}$ are complex constants, and $\gamma$ and $\beta$ are eigenvalues to be determined by the physical equations. Note that $\mathbf{b}_{c} \cdot \hat{z} \neq 0$, whereas $\mathbf{b}_{s} \cdot \hat{z}=0$, so that we have used the subscripts " $c$ " and " $s$ " to stand for compressional and shear in anticipation of having an equilibrium magnetic field in the $z$ direction.

From Eqs. (1), (3), and (4) it is easy to show the following relationships:

$$
\begin{aligned}
& \nabla \times \mathbf{b}_{c}=i k\left(1+\gamma^{2}\right)\left(\hat{z} \times \mathbf{b}_{c}\right), \\
& \nabla \times\left[\left(\nabla \times \mathbf{b}_{c}\right)=k^{2}\left(1+\gamma^{2}\right) \mathbf{b}_{c}\right], \\
& \nabla \times \mathbf{b}_{s}=\left(i b_{1} / k \beta\right)\left[\hat{z} \nabla^{2} J_{m}(\beta s)-i k \nabla J_{m}(\beta s)\right], \\
& \left(\nabla \times b_{s}\right)_{1}=i k \hat{z} \times \mathbf{b}_{s},
\end{aligned}
$$

and

$$
\nabla \times\left(\nabla \times \mathbf{b}_{s}\right)=k^{2}\left(1+\beta^{2}\right) \mathbf{b}_{s}
$$

where $\perp$ refers to the components perpendicular to the $z$ axis.

The MHD equations involve Ohm's law

$$
\mathbf{E}+\mathbf{U} \times \mathbf{B}=\eta \mathbf{J}
$$

and the equation of motion

$$
\rho \frac{d \mathbf{U}}{d t}=\mathbf{J} \times \mathbf{B}+\rho v \nabla^{2} \mathbf{U}
$$

where $\eta$ is the resistivity and $v$ is the viscosity.

By assuming an $\exp (-i \omega t)$ time dependence, taking the curl of Eq. (10), using Faraday's and Ampère's laws, and linearizing about an equilibrium where $\mathbf{U}_{0}=0$, $\mathbf{B}_{0}=B_{0} \hat{z}$, Eqs. (10) and (11) become

$$
-i \omega \mathbf{b}=B_{0} \nabla \times(\mathbf{u} \times \hat{z})+\frac{\eta}{\mu_{0}} \nabla^{2} \mathbf{b}
$$

and

$$
-i \omega \mathbf{u}\left(B_{0} / p \mu_{0}\right)(\nabla \times \mathbf{b}) \times \hat{z}+\nu \nabla^{2} \mathbf{u} .
$$

The $z$ component of Eq. (13) gives a simple viscous diffusion

$$
-i \omega u_{z}=\psi \nabla^{2} u_{z}
$$

and since $u_{z}$ occurs nowhere else in Eqs. (12) and (13) we may ignore $u_{z}$ from here on. We also note that $\nabla^{2} \mathbf{u}_{1}=\left(\nabla^{2} \mathbf{u}\right)_{1}$.

Let us now consider the term $(\nabla \times \mathbf{b}) \times \hat{z}$ in Eq. (13). From Eqs. (5) and (8) we see that, for both polarizations, this term is in the direction of $b_{1}$. Since the other terms (ignoring $u_{2}$ ) are both in the direction of $\mathbf{u}_{1}$, we see that $\mathbf{u}_{1}$ must be in the direction of $b_{1}$. Hence, let us assume that

$$
\mathbf{u}_{1}=-\lambda(\omega / k)\left(\mathbf{b}_{1} / B_{0}\right) \text {, }
$$

where $\lambda$ is a complex coefficient to be determined. From Eqs. (6), (9), and (15) we find that

$$
\nabla^{2} \mathbf{u}_{1}=\left(\lambda_{c} \omega k / B_{0}\right)\left(1+\gamma^{2}\right) \mathbf{b}_{c 1}
$$

for the compressional mode and

$$
\nabla^{2} \mathbf{u}_{1}=\left(\lambda_{\mathrm{s}} \omega k / B_{0}\right)\left(1+\beta^{2}\right) \mathbf{b}
$$

for a shear mode.

We now use these relations to solve the viscoresistive Alfvén wave equations. Using Eqs. (15)-(17) in Eq. (12) we see that all terms are proportional to $\boldsymbol{b}_{\boldsymbol{c}}$ or to $\mathbf{b}_{\mathrm{s}}$ so that 
Eq. (12) simply reduces to

$$
\lambda_{c, s}=1+i \kappa_{c, s}^{2} / S
$$

where $S=\mu_{0} V_{A}^{2} / \eta \omega$ is the magnetic Reynolds number, $\quad V_{\mathrm{A}}^{2}=B_{0}^{2} / \rho \mu_{0}$ is the Alfven velocity, $\kappa_{c}^{2}$ $=\left(1+\gamma^{2}\right) k^{2} V_{\mathrm{A}}^{2} / \omega^{2}$, and $\kappa_{\mathrm{s}}^{2}=\left(1+\beta^{2}\right) k^{2} V_{\mathrm{A}}^{2} / \omega^{2}$.

Let us now consider the compressional mode. Using Eqs. (5), (15), and (16) in Eq. (13) we find that all terms are now proportional to $\mathbf{b}_{c, 1}$, so Eq. (13) reduces to

$$
\lambda_{c}=\kappa_{c}^{2} /\left(1+i \kappa_{c}^{2} / R\right),
$$

where $R=V_{A}^{2} / v \omega$ is the fluid Reynolds number. Equating Eqs. (18) and (19) and realizing that both $R \gg 1$ and $S \gg 1$ we find a large root

$$
\kappa_{c}^{2}=-\mathrm{SR}
$$

and a small root

$$
\kappa_{c}^{2}=1+i / \mathrm{S}+i / \mathrm{R}
$$

The large root corresponds to highly evanescent modes and so may be ignored except for an infinitesimal layer at the plasma surface where it is needed to match boundary conditions prescribed at the surface. The small root describes modes in the bulk of the plasma and so we will restrict our attention to this root. Combining Eqs. (19) and (21) gives

$$
\lambda_{c}=1+i / S
$$

so that

$$
\mathbf{u}_{c \downarrow}=-\left(1+\frac{i}{\mathrm{~S}}\right) \frac{\omega}{k} \frac{\mathbf{b}_{c 1}}{B_{0}}
$$

and

$$
\gamma^{2}=\left(\frac{\omega^{2}}{k^{2} V_{\mathrm{A}}^{2}}-1\right)+i\left(\frac{1}{\mathrm{~S}}+\frac{1}{\mathrm{R}}\right) \frac{\omega^{2}}{k^{2} V_{\mathrm{A}}^{2}} .
$$

Following a similar strategy for the shear mode we find that $\left(\nabla \times \mathbf{b}_{s}\right) \times \hat{z}=i k \mathbf{b}_{s}$, which when substituted into Eq. (13) gives

$$
\lambda_{s}\left(1+i \kappa_{r}^{2} / R\right)=\kappa_{s}^{2}\left(1+\beta^{2}\right) .
$$

Solving for $\lambda$, and $\beta^{2}$ using Eqs. (18) and (25) we obtain

$$
\lambda_{\mathrm{s}}=1+[\mathrm{R} /(\mathrm{S}+\mathrm{R})]\left(k^{2} V_{\mathrm{A}}^{2} / \omega^{2}-1\right)
$$

and

$$
\beta^{2}=-1-i \frac{\left(1-\omega^{2} / k^{2} V_{\mathrm{A}}^{2}\right)}{(1 / \mathrm{S}+1 / \mathrm{R})},
$$

giving

$$
\mathbf{u}_{s}=-\left(1+\frac{\left(k^{2} V_{\mathrm{A}}^{2} / \omega^{2}-1\right)}{(1+\mathrm{S} / \mathrm{R})}\right) \frac{\omega}{k} \frac{\mathbf{b}_{\mathrm{s}}}{B_{0}} .
$$

Thus, Eqs. (3), (22)-(24), and (4), (25)-(28) provide analytic solutions for viscoresistive compressional and shear Alfvén waves in cylindrical geometry. These solutions are exact to order $\max (1 / \mathrm{S}, 1 / \mathrm{R})$.

\section{CURRENT DRIVE}

Let us now consider quasilincar current drive resulting from these modes. By quasilinear, we mean that when deriving Eqs. (3)-(28), we have not self-consistently taken into account the magnetic field produced by the dc current driven by the waves. We are interested in the $z$-directed dc current resulting from second-order beating of the modes, since this has the potential of driving a toroidal plasma current in a torus. From Eq. (10) this current is seen to be

$$
J_{z \mathrm{dc}}=(1 / 2 \eta) \operatorname{Re}\left(\mathbf{u}_{1} \times \mathbf{b}_{1}^{*} \cdot \hat{z}\right) .
$$

There are three possible configurations for Eq. (29): compressional modes beating with themselves, shear modes beating with themselves, and compressional modes beating with shear modes. We will consider each possibility separately.

(i) Compressional-compressional: ${ }^{27}$ Using Eq. (15), Eq. (29) becomes

$$
J_{z \mathrm{dc}}=\left(\omega / 2 \eta k B_{0}\right) \operatorname{Im}(\lambda) \operatorname{Im}\left(\mathbf{b}_{1} \times \mathbf{b}_{1}^{*} \cdot \hat{z}\right)
$$

since $\mathbf{b}_{1} \times \mathbf{b}_{1}^{*}$ is pure imaginary. From Eq. (3) we see that $b_{r}=-b_{z} J_{m}^{\prime}(\gamma s) / i \gamma$ and $b_{\theta}=-b_{z} m J_{m}(\gamma s) / \gamma^{2} s$, which with Eq. (22) gives

$$
\begin{aligned}
J_{z \mathrm{dc}}= & -\frac{m \omega\left|b_{z}\right|^{2}}{2 \eta k B_{0} \mathrm{~S}\left|\gamma^{2}\right| s} \\
& \times\left(\frac{J_{m}^{\prime}(\gamma s) J_{m}\left(\gamma^{*} s\right)}{\gamma^{*}}+\frac{J_{m}(\gamma s) J_{m}^{\prime}\left(\gamma^{*} s\right)}{\gamma}\right) .
\end{aligned}
$$

As expected, only $m=1$ modes are nonzero at $r=0$. Since our goal is on-axis drive, let us focus our attention to $m=1$ modes in the neighborhood of $r=0$. For small arguments, $J_{1}^{\prime}(\gamma s) \approx 1 / 2$ and $J_{1}(\gamma s) \approx \gamma s / 2$ so that Eq. (31) becomes

$$
\mu_{0} J_{z \mathrm{dc}}=-\omega \mu_{0}\left|b_{z}\right|^{2} / 4 \eta k B_{0} \mathrm{~S}\left|\gamma^{2}\right|
$$

which, using Eq. (24), can be expanded as

$$
\mu_{0} J_{\mathrm{zdc}}=-\frac{k\left|b_{z}\right|^{2}}{4 B_{0}} \frac{\left(\omega^{2} / k^{2} V_{\mathrm{A}}^{2}\right)}{\left[\left(\omega^{2} / k^{2} V_{\mathrm{A}}^{2}-1\right)^{2}+\left(\omega^{4} / k^{4} V_{\mathrm{A}}^{4}\right)(1 / \mathrm{S}+1 / \mathrm{R})^{2}\right]^{1 / 2}}
$$

The coefficient involving $\omega^{2} / k^{2} V_{\mathrm{A}}^{2}$ maximizes to $\mathrm{SR} /$ $(\mathrm{S}+\mathrm{R})$ at Alfvén resonance, i.e., when $\omega^{2} / k^{2} V_{\mathrm{A}}^{2}=1$, so that

$$
\max \left(\mu_{(0)} J_{z \mathrm{dc}}\right)=-\frac{k\left|b_{z}\right|^{2}}{4 B_{0}} \frac{\mathrm{SR}}{\mathrm{S}+\mathrm{R}} .
$$

At first sight, this looks like an attractive result because it provides a current proportional to the smaller of $R$ or $S$ both of which are large numbers.

However, it is necessary that the magnitude of the oscillating fields be small for the current drive scheme to be useful. Let us now examine these magnitudes. The equilibrium poloidal field associated with $J_{z \mathrm{dc}}$ is $B_{t_{\mathrm{tdr} \text { cin }}}=\mu_{0} J_{z \mathrm{dc}} r / 2$, the plasma safety factor is $q=r B_{0} / R B_{\theta_{\text {trincin }}}$, and the axial wave 
vector is related to the toroidal wave number $n$ by $k=n / R$, where $R$ is the major radius of the torus. From these relations, we see that

$$
\mu_{0} J_{z c_{\text {dincin }}}=2 B_{0} k / n q .
$$

Equating Eqs. (34) and (35) gives

$$
\frac{\left|b_{z}\right|}{B_{0}}=2\left[\frac{2}{n q}\left(\frac{1}{\mathrm{~S}}+\frac{1}{\mathrm{R}}\right)\right]^{1 / 2}
$$

so that $b_{z}$ is indeed small. Let us now evaluate the magnitude of $b_{\theta}$ to see if it is also small. From Eq. (3) we see that $b_{\theta}=-b_{z} / 2 \gamma$ or at Alfvén resonance

$$
b_{\theta}=-b_{z} / 2 \sqrt{i}(1 / \mathrm{S}+1 / \mathrm{R})^{1 / 2}
$$

so using Eq. (36)

$$
\left|b_{\theta}\right| / B_{0}=[2 / n q]^{1 / 2} \text {. }
$$

Since both $n$ and $q$ are of order unity, Eq. (38) indicates that the oscillating azimuthal magnetic field must be of the order of the dc axial field. A similar calculation shows that the oscillating radial field is of the same order. Such a large magnitude of oscillating magnetic field is unacceptable, since it would destroy equilibrium fiux surfaces and destroy particle confinement. Hence, we conclude that current drive by compressional Alfvén modes self-beating is not feasible.

(ii) Shcar-shear: From Eq. (26) we see that $J_{m}\left(\lambda_{s}\right)=0$ in which case Eq. (29) shows that the driven current is zero identically. Hence, there is no current drive at all due to shear waves beating with themselves.

(iii) Shear-compressional: In this situation Eq. (29) becomes

$$
\begin{aligned}
J_{z \mathrm{~d} c} & =(1 / 2 \eta) \operatorname{Re}\left(\mathbf{u}_{c} \times \mathbf{b}_{v}{ }^{*}+\mathbf{u}_{v} \times \mathbf{b}_{c}{ }^{*}\right) \cdot \hat{z} \\
& =-\left(\omega / 2 \eta k B_{0}\right) \operatorname{Re}\left[\left(\lambda_{c}-\lambda_{v}\right) \mathbf{b}_{c} \times \mathbf{b}_{*}^{*}\right] \cdot \hat{z},
\end{aligned}
$$

where we have used the fact that $\lambda$, is pure real. From Eqs. (22) and (26) we see that

$$
\lambda_{c}-\lambda_{s}=\frac{i}{S}-\frac{\mathrm{R}}{\mathrm{S}+\mathrm{R}}\left(\frac{k^{2} V_{\mathrm{A}}^{2}}{\omega^{2}}-1\right) \equiv \epsilon
$$

where $\epsilon$ is complex. Equation (27) shows that the shear wave is heavily damped radially unless

$$
\left|1-\omega^{2} / k^{2} V_{\mathrm{A}}^{2}\right| \ll 1 / \mathrm{S}+1 / \mathrm{R},
$$

in which case $|\epsilon|<S^{-1}$. Near $r=0$ we note (as before) that only $m=1$ modes survive, in which case

$$
\begin{aligned}
& b_{r c}=-\left(b_{z} / i \gamma\right) J_{i}(\gamma s) \approx-b_{z} / 2 i \gamma \\
& b_{i k}=-\left(b_{z} / \gamma^{2}\right) J_{1}(\gamma s) \approx-b_{z} / 2 \gamma \\
& b_{r}=\left(b_{1} / \beta s\right) J_{1}(\beta s) \approx b_{1} / 2
\end{aligned}
$$

and

$$
b_{t_{\mathrm{s}}}=i b_{1} J_{i}(\beta s) \approx i b_{1} / 2
$$

Thus, Eq. (39) becomes

$$
J_{z \mathrm{dc}}=-\left(\omega / 4 \eta k B_{0}\right) \operatorname{Re}\left(\epsilon b_{z} b_{i}^{*} / \gamma\right)
$$

which is clearly at a maximum when $|\gamma|$ is at a minimum. Equation (24) shows that $|\gamma|$ is at a minimum when $\omega^{2} / k^{2} V_{\lambda}^{2}=1$, in which case

$$
\chi_{\mathrm{nm}}=\sqrt{i}(1 / \mathrm{S}+1 / \mathrm{R})^{1 / 2} .
$$

Thus Eq. (42) becomes

$$
\mu_{i p} J_{z \mathrm{~d} s} \sim-\frac{k}{4 B_{0}} \operatorname{Re}\left(\frac{b_{z} b_{1}^{*}}{(1 / \mathrm{S}+1 / \mathrm{R})^{1 / 2}}\right) .
$$

Equating Eqs. (35) and (44), and using Eq. (37) gives

$$
\left|b_{c \theta} b_{* 1}^{*}\right| / B_{0}^{2} \sim 4 / n q \text {. }
$$

Again, since $n q$ is of order unity, we find that the oscillating fields are of the order of the equilibrium toroidal field. Hence, current drive by shear waves beating with compressional waves is also not feasible.

\section{SUMMARY}

We have shown that in the quasilinear $\mathrm{MHD}$ regime with both resistivity and viscosity taken into account, it is not possible to drive useful (i.e., significant on-axis) current using any combination of the modes existing in this regime. This does not mean that low frequency current drive is necessarily impossible, it simply means that if such a scheme does exist, it must involve physical phenomena other than those contained in the classical MHD model discussed here. Obvious candidates for such added phenomena are the following.

(i) The Hall term (as in the rotamaks), in which case Ohm's law becomes

$$
\mathbf{E}+\mathbf{U} \times \mathbf{B}-(1 / n e) \mathbf{J} \times \mathbf{B}=\eta \mathbf{J},
$$

where here $n$ is the electron density. The Hall term $\mathbf{J} \times \mathbf{B} /$ ne may be shown to be of order $\omega / \omega_{c i}$.

(ii) A full nonlinear treatment, where the wave equations are self-consistently solved for in the presence of the driven current (this ought to lead to some aspects of the nonlinear skin effect that is so important to the rotamak).

(iii) Minority species ion cyclotron damping kinetic effects as discussed by Chan, Ohkawa, and Miller. ${ }^{7}$ However, due to the localized nature of cyclotron resonances, it may be difficult for these effects to provide a bulk, rather than localized, current.

\section{ACKNOWLEDGMENT}

This work was supported by U.S. Department of Energy Grant DE-FG03-86ER53232.

\footnotetext{
'Proceedings of the Eighth Topical Conference on Radio Frequency Power in Plasmas, edited by R. McWilliams (AIP, New York, 1989), Chap. 2; see also L. H. Sverdrup and P. M. Bellan, Phys. Rev. Lett. 59, 1197 (1987) and references therein.

See Ref. 1. Chap. 1

'R. McWilliams, D. P. Sheehan, N. S. Wolf, and D. Edrich, in Ref, 1, p. 162.

${ }^{+} T$. Ohkawa. Conmments Plasma Phys. Controlled Fusion 12, 165 (1989).

${ }^{5}$ R. R. Mett and J. A. Tataronis, Phys. Rev. Lett. 63, 1380 (1989).

"J. B. Taylor, Phys, Rev, Lett. 63, 1384 (1989).

'V. S. Chan, R. L. Miller, and T. Ohkawa, Phys. Fluids B 2, 944 (1990)

${ }^{*}$ T. H. Jensen and M. S. Chu, Phys. Fluids 27, 2881 (1984).

"M. K. Bevir and J. W. Grey, in Proceedings of the Reverse Field Pinch Theory Workshop (Los Alamos Scientific Laboratory, Los Alamos, NM, 1981). Vol. 3. paper A-3.

${ }^{113}$ K. F. Schoenberg, C. J. Buchenauer, R. S. Massey. J. G. Melton, R. W. Moses, R. A. Nebel, and J. A. Phillips, Phys. Fluids 27, 548 (1984).

"P. M. Bellan. Phys. Fluids 27, 191 (1984).

'P. M. Bellan. Phys. Rev. Lett. 57, 2383 (1986).

"M. A. Schalit and P. M. Bellan. Phys. Fluids B 1, 2533 (1989).

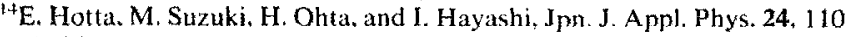
(1985).
} 
${ }^{1}$ K. F. Schoenberg, J. C. Ingraham, C. P. Munson, P. G. Weber, D. A. Baker, R. F. Gribble, R. B. Howell, G. Miller, W. A. Reass, A. E. Schofield, S. Shinohara, and G. A. Wurden, Phys. Fluids 31, 2285 (1988).

"P. M. Bellan, Nucl. Fusion 29, 78 (1989).

'I. R. Jones, Comments Plasma Phys. Controlled Fusion 10, 115 (1986).

"*W. N. Hugrass, J. Plasma Phys. 28, 369 (1982).

${ }^{14}$ G. A. Collins, G. Durance, G. R. Hogg, J. Tendys, and P. A. Watterson, Nucl. Fusion 28, 255 (1988).

2"P. M. Bellan, Plasma Phys. Controlled Fusion 31, 879 (1989).

"M. Dutch and A. L. McCarthy, Plasma Phys. Controlled Fusion 28, 695 (1986).

"H. A. Blevin and P. C. Thonemann, Nucl. Fusion, Suppl., Part I, 55
(1962).

${ }^{23}$ K. V. Khodataev, V. P. Shimchuk, and V. A. Shchelokov, Nucl. Fusion 10, 37 (1970).

${ }^{24}$ N. A. Borzunov, N. Ya. Kuz'mina, I. Kh. Nevyazhskii, S. M. Osovets, Yu. F. Petrov, B. I. Polyakov, I. A. Popov, K. V. Khodataev, and V. P. Shimchuk, Sov. Phys. Dokl. 8, 914 (1964) [Dokl. Akad. Nauk SSSR 152, 581 (1963).

${ }_{25}^{25}$ H. R. Lewis and P. M. Bellan, J. Math. Phys. 31, 2592 (1990).

${ }^{26}$ H. K. Moffatt, Magnetic Field Generation in Electrically Conducting Fluids (Cambridge U. P., London, 1978), pp. 21,22.

${ }^{27}$ Discussed for the case of no viscosity in M. A. Schalit, Ph.D. thesis, California Institute of Technology, 1989. 